\title{
Repression of human activation induced cytidine deaminase by miR-93 and miR-155
}

Glen M Borchert, Nathaniel W Holton and Erik D Larson*

\begin{abstract}
Background: Activation Induced cytidine Deaminase (AID) targets the immunoglobulin genes of activated B cells, where it converts cytidine to uracil to induce mutagenesis and recombination. While essential for immunoglobulin gene diversification, AID misregulation can result in genomic instability and oncogenic transformation. This is classically illustrated in Burkitt's lymphoma, which is characterized by AID-induced mutation and reciprocal translocation of the $c-M Y C$ oncogene with the IgH loci. Originally thought to be B cell-specific, AID now appears to be misexpressed in several epithelial cancers, raising the specter that AID may also participate in non-B cell carcinogenesis.

Methods: The mutagenic potential of AID argues for the existence of cellular regulators capable of repressing inappropriate AID expression. MicroRNAs (miRs) have this capacity, and we have examined the publically available human AID EST dataset for miR complementarities to the human AID 3'UTR. In this work, we have evaluated the capacity of two candidate miRs to repress human AID expression in MCF-7 breast carcinoma cells.

Results: We have discovered moderate miR-155 and pronounced miR-93 complementary target sites encoded within the human AID mRNA. Luciferase reporter assays indicate that both miR-93 and miR-155 can interact with the $3^{\prime} U T R$ of AID to block expression. In addition, over-expression of either miR in MCF-7 cells reduces endogenous AID protein, but not mRNA, levels. Similarly indicative of AID translational regulation, depletion of either miR in MCF-7 cells increases AID protein levels without concurrent increases in AID mRNA.

Conclusions: Together, our findings demonstrate that miR-93 and miR-155 constitutively suppress AID translation in MCF-7 cells, suggesting widespread roles for these miRs in preventing genome cytidine deaminations, mutagenesis, and oncogenic transformation. In addition, our characterization of an obscured miR-93 target site located within the AID $3^{\prime} U T R$ supports the recent suggestion that many miR regulations have been overlooked due to the prevalence of truncated $3^{\prime} U T R$ annotations.
\end{abstract}

Keywords: AICDA, AID, CSR, hypermutation, microRNA, miR-93, miR-155, SHM, UTR, 3'UTR

\section{Background}

In antigen activated B cells the Activation Induced cytidine Deaminase (AID) protein is required for initiating mutagenesis and recombination of the immunoglobulin (Ig) genes to promote immunity. AID is a cytidine deaminase that converts single-stranded genomic cytidine into uracil, and its activity is pronounced at the Ig variable (V) and switch (S) regions [1-5]. While spontaneously generated uracil in the genome is faithfully corrected as a part of normal DNA repair [6], AID-

\footnotetext{
* Correspondence: elarson@ilstu.edu

School of Biological Sciences, Illinois State University, Normal, IL 61790-4120,
} USA

\section{Biomed Central}

induced uracils at the Ig loci are mutagenically resolved by multiple DNA repair factors [4,7]. Although AID targets the Ig loci in activated B cells, activity at other genomic sites has the potential to create oncogenic DNA damage. Indeed, transcribed genes across the genome have now been found to undergo AID deamination (reviewed by $[8,9]$ ), and recently, deep sequencing of AID-ChIPed template revealed broad AID-associations with expressed loci [10]. In addition, AID-dependent DNA breaks were recently identified within multiple types of repetitive elements [11] suggesting a broad ability for AID to target genomic regions harboring singlestranded character. 
AID misexpression results in DNA damage that promotes cancer [11-16]. In lymphoid cells improper AID expression has been connected with mutations at $c$ MYC, PIM1, RhoH and PAX5 oncogenes, promoting diffuse large B cell lymphoma [17]. Similarly, Burkitt's lymphoma is characterized by AID-induced mutation and a reciprocal translocation between the $c-M Y C$ proto-oncogene and the IgH loci $[9,18-20]$. While $A I D$ expression was initially thought to be $B$ cell-specific, recent evidence indicates AID may promote the development of various non-lymphoid oncologies. In gastric cancer, the upregulation of $A I D$ leads to point mutations and copy number alterations of CDKN2A and CDKN2B tumor suppressor genes [21]. In the same manner, AID misexpression in human colonic cells increases the mutation rate of TP53 by $\sim 10$-fold [22]. In addition, AID is expressed at various levels in $\sim 1 / 3$ of primary lung cancers [23] and in numerous breast cancer cell lines [24] (where AID misexpression may be due in part to observations that estrogen is capable of inducing AID expression $>20$ fold [25]). Since errant AID activity can introduce significant genomic damage, the maintenance of genome stability outside of the activated B cell environment likely depends upon multiple molecular AID restraints.

Recent evidence suggests that one level of AID regulation comes from microRNAs (miRs). These short, $\sim 20$ nt, noncoding RNAs can regulate networks of genes and function by repressing translation or directing mRNA destruction through partial sequence complementarity to 3' untranslated regions (3'UTRs) of mRNAs [26,27]. MiRs are key regulators of cellular differentiation, proliferation and apoptosis, and aberrant miR expression has been associated with a myriad of human diseases, including cancer (reviewed by [28]). A subset of miRs typically misexpressed in malignancy (oncomiRs) can function as oncogenes or tumor suppressors with impacts on cellular transformation and metastasis. One such oncomiR, miR-155, represses murine $A I D$, and the disruption of the miR-155 recognition site or miR-155 itself results in increased AID-induced $c-M Y C$ translocation [29] and BCL6 mutagenesis [30]. This may provide one explanation for why disruption of miR-155 is associated with Burkitt's lymphoma [31] and suggests the existence of parallel miR repressions in humans.

In order to better define the regulators of human AID in cells other than antigen-activated B cells, we examined the 3'UTR of human AID for miR complementarities. Consistent with the role of miR-155 in regulating $A I D$ in mice $[29,30]$, we find a moderate miR-155 site in human $A I D$. Surprisingly, we also find a previously uncharacterized, yet exceptional, complementarity to miR-93 in the AID 3'UTR. We show both of these miRs interact with the AID 3'UTR in the cell to regulate its translation, and that loss of either miR results in increased AID protein levels. Based on the involvement of AID in generating oncogenic genome mutations, our results suggest that miR-93 and miR-155 act as dual genome sentries to prevent errant translation of $A I D$ mRNA.

\section{Methods}

\section{Reagents and cell lines}

Oligonucleotide sequences are detailed in Additional File 1, Table S1. Human embryonic kidney (HEK293) cell line was obtained from GenLantis (San Diego, CA), Burkitt's lymphoma (Ramos) and breast cancer (MCF-7) cells were both purchased from the American Type Culture Collection, (ATCC, Manassas, VA). HEK293 and MCF-7 cells were cultured in MEM (Mediatech, Herndon, VA) supplemented with $10 \%$ fetal bovine serum (Hyclone, Logan, UT). Ramos cells were cultured in RPMI (Mediatech) supplemented with 10\% fetal bovine serum (Hyclone, Logan, UT). All tissue culture media were supplemented with $25 \mathrm{mg} / \mathrm{ml}$ streptomycin and 25 I.U. penicillin (Mediatech). Cells were cultured in a humidified atmosphere with $5 \% \mathrm{CO} 2$ at $37^{\circ} \mathrm{C}$. For luciferase assays, HEK293 s were cultured in MEM (10\% FBS and 1\% PS) in 12-well plates. At 90\% confluency, cells were transfected following the Lipofectamine 2000 (Invitrogen, Carlsbad, CA) protocol. At 35 h, existing media was replaced with $1 \mathrm{ml}$ fresh media. For miR expression assays, MCF-7 s were cultured in MEM (10\% FBS and 1\% PS) in 6-well plates. At 90\% confluency, cells were transfected following the Lipofectamine 2000 (Invitrogen) protocol and harvested after $48 \mathrm{~h}$. For miR sponge assays, MCF-7 s were cultured in MEM (10\% FBS and 1\% PS) in 6-well plates. At 70\% confluency, cells were transfected following the Lipofectamine 2000 (Invitrogen) protocol. At $48 \mathrm{~h}$, existing media was replaced with $2 \mathrm{ml}$ fresh media and cell transfections were repeated as initially performed. Cells were then harvested after an additional $48 \mathrm{~h}$ culture.

\section{Luciferase assays}

At $36 \mathrm{~h}$ post transfection, cells were scraped from well bottoms and transferred to $1.5 \mathrm{ml}$ Eppendorf tubes. Eppendorfs were centrifuged at 2000 RCF for $3 \mathrm{~min}$, followed by supernatant aspiration and cell resuspension in $300 \mu \mathrm{l}$ of PBS. Cells were lysed by freeze thaws and debris removed by centrifuging at $3000 \mathrm{RCF}$ for $3 \mathrm{~min} .50$ $\mu \mathrm{l}$ of supernatant was transferred to a 96-well MicroLite plate (MTX Lab Systems, Vienna, VA) then firefly and Renilla luciferase activities measured using the Dual-glo Luciferase $^{\circledR}$ Reporter System (Promega, Madison, WI) and a 96-well plate luminometer (Dynex, Worthing, West Sussex, UK). RLUs were calculated as the quotient of Renilla/firefly RLU and normalized to mock. 


\section{Western blotting}

MCF-7 cells (at $\sim 1 \times 10^{6}$ cells $/ \mathrm{ml}$ ) were pelleted by centrifugation, existing media removed, and cells resuspended in SDS lysis buffer containing protease inhibitors and transferred to $1.5 \mathrm{ml}$ Eppendorf tubes. Proteins were electrophoresed through a 4-12\% SDSpolyacrylamide gradient gel (Invitrogen) and transferred to immobilon-P PVDF membranes (Millipore, Temecula, CA). Membranes were blocked for 1 hour in $5 \%$ $(\mathrm{w} / \mathrm{v})$ nonfat milk in phosphate-buffered saline containing $0.05 \%$ Tween 20, washed, and incubated with primary antibody overnight at $4{ }^{\circ} \mathrm{C}$ using the following dilution: anti-AID (Santa Cruz Biotechnology, Santa Cruz, CA, sc-25620) - 1:500 and anti-PCNA (Santa Cruz) - 1:10000. Membranes were washed and incubated with secondary Abs: HRP conjugated goat antimouse and goat anti-rabbit (Invitrogen) at 1:10000 dilution. Immunoreactive bands were visualized with ECL Plus (GE, Piscataway, NJ) and signals were detected by using the Storm 840 PhosphorImager and IMAGEQUANT software (GE).

\section{Vector construction}

Unless otherwise indicated, PCR amplifications were performed in $40 \mu \mathrm{l}$ reactions at standard concentrations (1.5 mM $\mathrm{MgCl}_{2}, 0.2 \mathrm{mM}$ dNTP, $1 \times$ Biolase PCR buffer, 0.5 U Taq (Bioline USA, Inc., Randolph, MA), $0.5 \mathrm{uM}$ each primer) and using standard cycling parameters $\left(94^{\circ}\right.$ $\mathrm{C}-3 \mathrm{~min},\left(94^{\circ} \mathrm{C}-30 \mathrm{~s}, 55^{\circ} \mathrm{C}-30 \mathrm{~s}, 72^{\circ} \mathrm{C}-60 \mathrm{~s}\right) \times 30$ cycles, $72^{\circ} \mathrm{C}-3 \mathrm{~min}$ ) then cloned into Topo PCR 2.1 (Invitrogen). RT-PCRs were performed at $65^{\circ} \mathrm{C}$ using MonsterScript Reverse Transcriptase (\#MSTA5110, Epicentre, Madison, WI) and gene specific or random nonamer primers. Resultant amplicons were cloned into Topo PCR 2.1 and sequenced. Antisense reporter, Ctl 3'LR, was constructed by oligonucleotide primer extension ( 25 cycles with $10 \mathrm{~s}$ extensions) with primers containing 5' Xho-I and 3' Spe-I restriction enzyme sites immediately flanking sequences perfectly complementary to mature siLacZ. Antisense reporter, AID 3'LR, was constructed by standard PCR with primers containing 5' Xho-I and 3' Spe-I restriction enzyme sites. Following digestion, amplicons were ligated into the Renilla luciferase 3'UTR of psiCheck2 (Promega) vector linearized with Xho-I and Spe-I. The presence of an independently transcribed firefly luciferase in these reporters allowed normalization for transfection efficiency. Sponge expression constructs were generated by concatamerizing PCR using the primers indicated in Additional File 1, Table S1. Resulting amplicons were separated on a $1 \%$ agarose gel and a band excised from the appropriate lane at $\sim 400 \mathrm{bp}$. Gel extractions were cloned into Topo PCR 2.1 and sequenced. Concatamers were next excised from Topo PCR 2.1 and cloned into the pEGFP expression vector (Clontech, Mountain View, CA) using BamHI and Not I restriction sites.

\section{Results}

The human AID 3'UTR contains sequences complementary to miR-93 and miR-155

In light of the evidence supporting $A I D$ regulation by miR-155 in mouse showing miR-155 regulates AID translation and c-myc translocation $[29,30]$, we asked if human $A I D$ is regulated by similar microRNA interactions. Computational analysis of the human AID 3'UTR revealed a human miR-155 target site, consistent with studies in mice $[29,30]$. To our surprise, we also found pronounced complementarity between the AID 3'UTR and miR-93 (Figure 1A). This miR-93 target site is markedly more extensive than the majority of characterized miR::mRNA interactions containing only a single, centrally located $4 \mathrm{bp}$ mismatch. Strikingly, we find the

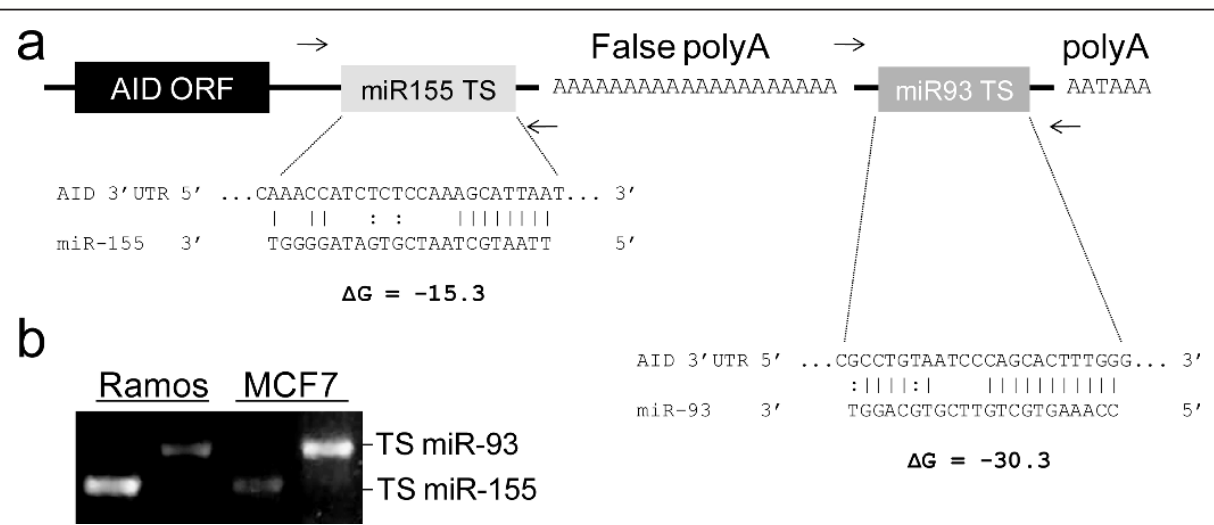

Figure 1 The AID 3'UTR. (A) Cartoon of AID 3'UTR miR target sites (TS) and internal (False) polyA (not to scale) (DNA sequence shown) MicroRNA alignments are shown with corresponding binding affinities ( $\mathrm{kcal} / \mathrm{mol})$. Vertical lines indicate complementary base pairing. Dotted vertical lines indicate G:U base pairing. PolyA, AATAAA eukaryotic polyadenylation signal. Arrows indicate RT-PCR primer sets for confirmation of expression. (B) RT-PCR to test expression of the AID 3'UTR miR-155 and miR-93 target site (TS) sequences in Ramos and MCF-7 cells. 
predicted binding affinity between miR-93 and the AID 3'UTR $(-30.3 \mathrm{kcal} / \mathrm{mole})$ to be nearly double that of miR-155 (-15.3 kcal/mole) (Figure $1 \mathrm{~A})$ suggesting a strong post-transcriptional regulatory relationship. Further analysis of the human AID 3'UTR revealed an internal $20 \mathrm{nt}$ stretch of adenosines (sufficient for cDNA poly-T priming) separating the miR-155 and miR-93 target sites (Figure 1A). This false polyA is located $\sim 1,000$ nt 3' of the AID stop codon and does not correspond to the full length cDNA polyadenylation as: (1) it is not preceded by the canonical 5' AAUAAA poly adenylation signal or other recognizable alternative poly adenylation signal, and (2) the miR-93 target site is expressed in both Ramos and MCF-7 cells (Figure 1B). Importantly, examination of publically available EST datasets confirms this internal polyA stretch has repeatedly served as an oligo-dT priming site for EST reverse transcriptase leading to the improper annotation of a truncated AID cDNA (for example see NCBI accessions GI:33871601 and GI:50496022). We conclude that the human AID 3'UTR contains miR-155 and miR-93 recognition sites, which are separated by a 20 nucleotide adenosine repeat upstream from the true polyA sequence.

\section{MiR-93 and miR-155 interact with the AID 3'UTR to inhibit its expression}

The identification of miR-93 and miR-155 binding sites in the 3'UTR of human AID strongly suggests post-transcriptional regulatory interactions. Therefore, we next asked if miR-93 and miR-155 were capable of regulating the AID 3'UTR using standard luciferase reporter assays. Three expression constructs incorporating an endogenous human microRNA Alu promoter were created by cloning the endogenous miR-517a genomic locus and then replacing the miR-517a hairpin with the miR-93, miR-155 and shLacZ hairpins (pAL-93, pAL-155, and pAL-1 respectively)(Figure 2A), as described [32]. We also generated 3'UTR reporter constructs to test AID:: miR regulatory interactions. These reporters (AID 3'LR and Ctl 3'LR) produce Renilla luciferase transcripts tailed with the AID 3'UTR and shLacZ target sequence respectively (Figure $2 \mathrm{~B}$ ). Co-transfection of human embryonic kidney (HEK293) cells with pAL-93 and AID 3'LR constructs resulted in $~ 80 \%$ knockdown of Renilla luciferase intensity, whereas co-transfection of pAL-155 and AID 3'LR constructs resulted in $\sim 60 \%$ knockdown of Renilla luciferase intensity at a 4:1 transfection ratio (Figure 2C). Similarly and as expected, co-transfection of pAL-1 and Ctl 3'LR resulted in a reduction of Renilla luciferase intensity with $\sim 95 \%$ knockdown (Figure 2D). In contrast and as expected, neither the co-transfection of pAL-1 with AID 3'LR nor co-transfection of either miR construct with Ctl 3'LR resulted in silencing (Figure $2 \mathrm{C}-\mathrm{D})$. To further ensure the repressions of AID 3'LR

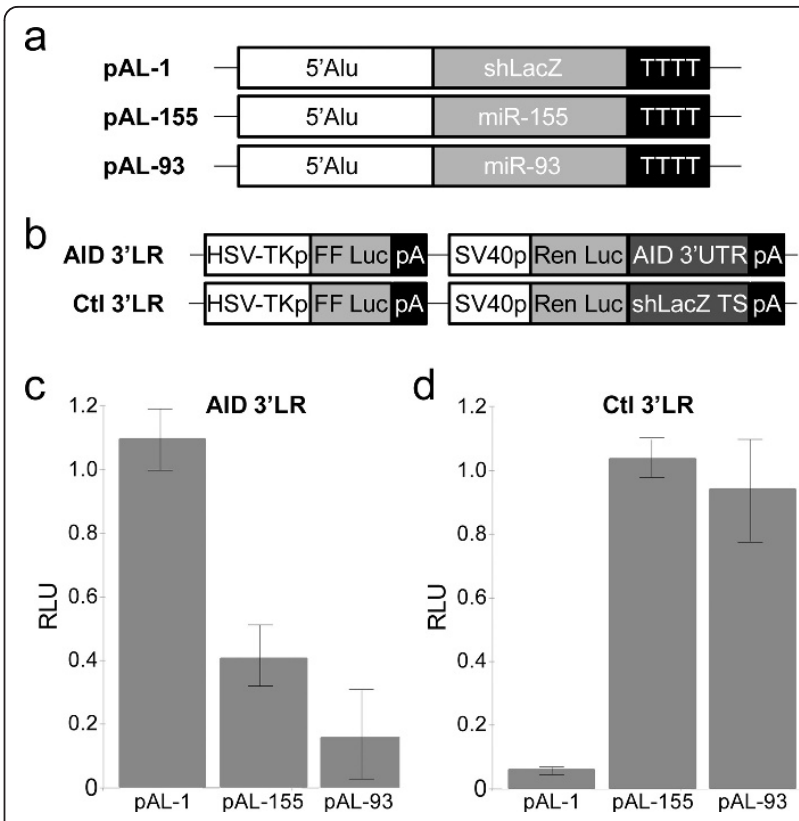

Figure 2 The AID 3'UTR is recognized by miRs -93 and -155 . (A) Diagrams of miR and siRNA expression vectors. 5'Alu corresponds to a genomic fragment containing the miR-517a upstream Alu promoter [32]. In PAL-1, shLacZ replaces the miR-517a hairpin. In PALs-155 and -93, the pre-miR-155 and -93 hairpins replace the miR-517a hairpin respectively. TTT, RNA polymerase III termination signal. (B) Diagrams of luciferase reporters. The AID 3'UTR luciferase reporter (AID 3'LR) and control $3^{\prime} U T R$ luciferase reporter (CtI $3^{\prime} \mathrm{LR}$ ) were generated respectively by placing an AID $3^{\prime} U T R$ sequence containing both the miR-155 and miR-93 target sites and a specific region of LacZ previously shown to be targeted by the PAL-1 shLacZ hairpin in a multiple cloning site in the Renilla luciferase 3'UTR [32]. HSV-TKp, herpes simplex virus thymidine kinase promoter; SV40p, simian virus 40 promoter; FF Luc, firefly luciferase; Ren Luc, Renilla luciferase; pA, poly(A). (C) AID 3'LR is specifically repressed by both miR-93 and miR-155 in 293 transient transfections. Luciferase assays $(n=4)$ of HEK293 lysates after transfection of AID 3'LR and either pAL-1, pAL-155 or pAL-93. Transfections were normalized to AID 3'LR alone. RLU, relative light units. (D) Ctl 3'LR is specifically repressed by the ctl (pAL-1) in 293 transient transfections. Cotransfections done as in (C), except Ctl 3'LR replaced AID 3'LR.

by pAL-93 and pAL-155 were due to specific interactions between miRs -93 and -155 and their proposed target sites in the AID 3'UTR, we constructed two additional luciferase reporters whose 3'UTRs consisted of: (1) the two target sites with intervening sequence removed and (2) scrambled versions of the two target sites with intervening sequence removed. Again, we found co-transfection of human embryonic kidney (HEK293) cells with pAL expression constructs and a luciferase reporter containing intact miR target sites resulted in significant repression of Renilla luciferase in contrast to control transfections of scrambled miR target site reporters, which were not repressed (Additional File 2, Figure S1). Therefore, miR-93 and miR-155 
facilitate molecular interactions with the AID 3'UTR sufficient to repress translation.

\section{MiR-93 and miR-155 can repress endogenous AID}

Interactions between miR-93, miR-155 and the AID 3'UTR within the context of our reporter assays (Figure 2C-D) strongly support the involvement of both miRs in $A I D$ translational regulation. If so, we anticipated that if miR-93 and/or miR-155 were present in AID expressing cells they could suppress endogenous AID protein levels. We therefore asked if miR-93 and miR-155 are present in two cell lines known to express $A I D$, Ramos and MCF-7. Ramos is a B cell line that undergoes constitutive AID-induced somatic hypermutation [33], and MCF-7 is a breast carcinoma that misexpresses AID [24]. Using standard RT-PCR analysis, we found miR -93 and -155 transcripts are expressed in both cell lines (Additional File 3, Figure S2), consistent with the ubiquitous expression documented for both of these miRs [34]. In order to determine if endogenous AID can be repressed by increased miR-93 and/or miR-155 expression, we introduced pAL miR over-expression plasmids (Figure 2A) into MCF-7 cells [32]. Importantly, overexpression of miR-93, miR-155, or an AID-targeting siRNA (shAID) in MCF-7 cells each reduced AID protein levels between 70 and $90 \%$, whereas overexpression of the pAL-1 control did not significantly alter $A I D$ expression (Figure 3A). To ensure results were the consequence of miR regulation, we confirmed the miR-93 repression of $A I D$ was dose-dependent (Additional File 4, Figure S3) and isolated total RNA from each of our transfections to monitor AID mRNA levels by quantitative PCR. Strikingly, while shAID transfection averaged a $92 \%$ reduction of $A I D$ mRNA levels, neither pAL-93 nor pAL-155 transfection resulted in significant reduction of AID mRNA (Figure 3B) confirming that the observed reductions in AID protein levels following their transfections are mediated through translational repression. To summarize, our results demonstrate that the decreases in AID protein upon shAID transfection were due to message degradation, whereas pAL-93 and pAL-155 overexpression resulted in miR-mediated translational repression.

\section{Endogenous miR-93 and miR-155 restrain AID protein translation}

Over-expression of miR-93 and miR-155 repressed the expression of endogenous $A I D$, supporting the model that both miRs contribute to post-transcriptional regulation of AID. To be consistent with that hypothesis, the loss of cellular miR-93 or miR-155 should result in higher $A I D$ expression. Therefore, we depleted miR-93 and miR-155 individually and then monitored changes to AID protein levels by western blotting. To

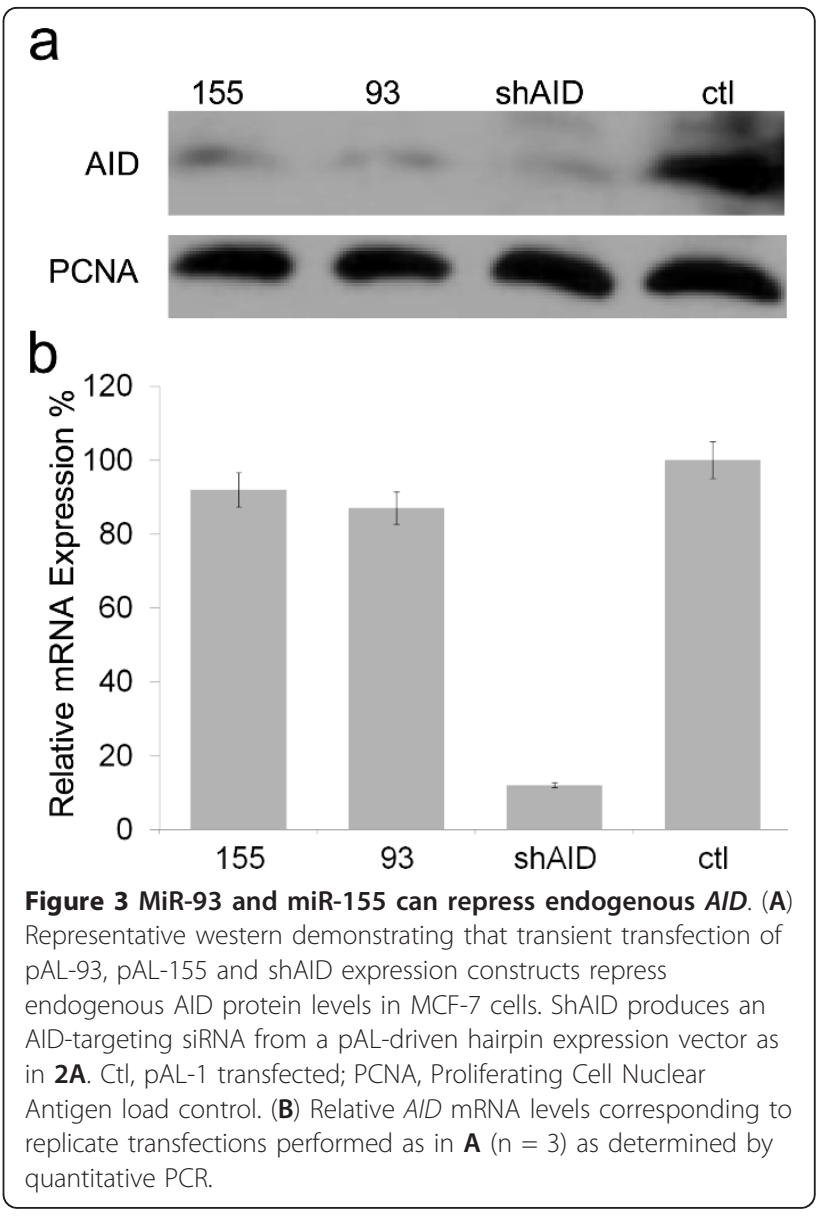

achieve this, we constructed miR sponge transgenes for miR-93 and miR-155 that function by competitively inhibiting miR activity, as previously described $[35,36]$. Our miR sponge transgenes produce RNAs containing $\sim 10$ copies of the miR-93 or miR-155 AID 3'UTR complementary binding sites (Figure 4A). Individual miR sponges or a negative control sponge transgene were transiently transfected in MCF-7, and relative AID protein levels measured by western. MCF-7 cells expressing either miR-93 or miR-155 sponges showed clear dose-responsive increases in AID protein levels, whereas control transfections did not significantly alter AID protein expression (Figure 4B). To ensure these findings were the consequence of restricted miR activity, we isolated total RNA from each of our transfections and examined AID mRNA levels by quantitative PCR. We found AID mRNA levels largely unaffected by transfection of sponge constructs further supporting endogenous roles for these miRs in the restraint of AID translation (Figure $4 \mathrm{C})$. We conclude that one function of endogenous miR-93 and miR-155 expression is to restrict the translation of AID mRNA. 


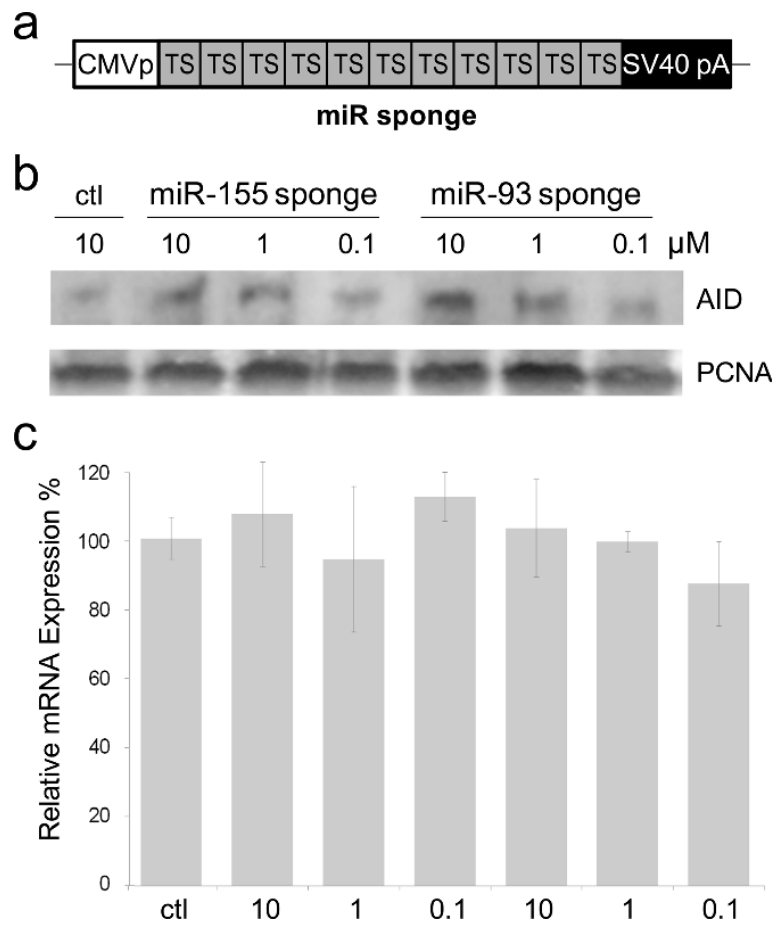

Figure 4 Endogenous miR-93 and miR-155 restrain AID protein expression. (A) Diagram of miR sponge expression vector. TS indicates an AID 3'UTR miR target site detailed in 1A. The miR-93 sponge produces a polyadenylated concatamer of 11 individual 93 TSs. Analogously designed, the miR-155 sponge contains 10 individual miR-155 TSs, and the ctl sponge contains 14 individual shLacZ targets. CMVp, immediate early cytomegalovirus promoter; SV40pA, simian virus 40 polyadenylation signal. (B) Representative western demonstrating that transient transfection of MCF-7 cells with the miR-93 or miR-155 sponges results in dose responsive increases in AID protein levels as compared to control. Ctl, shLacZ target site sponge transfected; PCNA, Proliferating Cell Nuclear Antigen. (C) Relative AID mRNA levels corresponding to replicate transfections performed as in $\mathbf{B}(n=3)$ as determined by quantitative PCR.

\section{Discussion}

\section{Model for AID repression}

We have shown that miR-93 and miR-155 have the capacity to repress AID translation and do so in the cellular context. Overexpression of miR-93 or miR-155 reduced AID protein levels in MCF-7 breast cancer cells (Figure 3A), whereas depletion of either endogenous miR-93 or miR-155 resulted in increased AID translation (Figure 4) directly connecting miR expression with changes in AID protein concentration. Based on our findings, we propose a model for the relationship between miRs -93 and -155 and $A I D$ regulation in maintaining genomic integrity (Figure 5). In cellular circumstances where high levels of AID transcription exist (such as antigen activated B cells or some established cancers) $A I D$ mRNA levels overwhelm miR controls. In

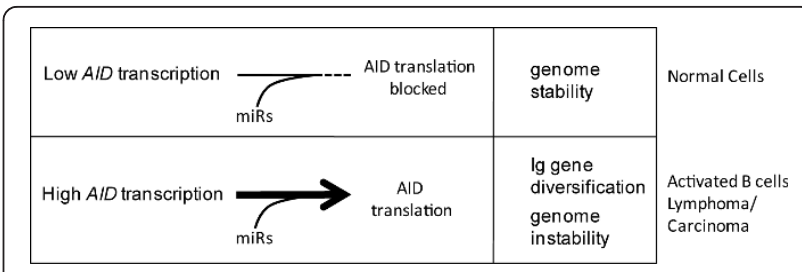

Figure 5 Model of miR-93 and miR-155 regulation of AID mediated genomic instability. In normal cells, top, genome stability is maintained by inhibition of AID translation by miR-93 and/or miR-155 when AID mRNA levels are low. Bottom, in antigen activated $B$ cells or when misregulated, levels of AID mRNA exceed the capacity for miR translational repression. AID translation results in AID protein levels that support Ig gene diversification,

mutagenesis and genome instability.

these situations, endogenous miR-155 and miR-93 have negligible influence on AID protein levels. In contrast, miR-93 and miR-155 can greatly reduce the potential for errant $A I D$ expression which protects the genome from unwanted cytidine deamination and mutation by restricting AID protein production (Figure 5). This may be particularly important during S-phase when replication transiently denatures the DNA. Conspicuously, miR-93 is intronically encoded within the minichromosome maintenance 7 (MCM7) gene, an essential replication licensing factor, and precursor miR-93 abundance tracks with MCM7 expression levels [37,38]. It is therefore tempting to speculate that domestication of the miR-93 microRNA along with the corresponding AID 3'UTR site occurred as a response to the selective pressure for maintaining genome fidelity during replication.

\section{Disruptions of miR-155 and miR-93 are associated with AID-induced oncogenesis}

Highlighting the importance of tightly controlled $A I D$ expression, novel $A I D$ regulations continue to be described. In 2008, miR-155 was identified as an important repressor of AID translation in mice, and loss of this regulation is lymphomagenic $[29,30]$. Our data now demonstrate directly that expression of endogenous miRs -93 and -155 represses AID translation in MCF-7 breast cancer cells. While a direct correlation between miR-93 activity and AID-induced oncogenesis remains to be described, miR-93 perturbations have been found to enhance cell survival, possess oncogenic activities, and augment tumor growth through regulating integrin$\beta 8$, the tumor suppressor gene FUS1, the Cdk inhibitor p21, and tumor protein 53-induced nuclear protein 1 (TP53INP1) [39-42]. Our results suggest that in addition to these known oncogene regulations, loss of miR-93 (or miR-155) may permit AID upregulation and mutator phenotypes in non-B cell oncologies (e.g. breast, colon, stomach and lung [21-24]). However, it is possible that 
additional miR AID regulations may still be described, and our research does not seek to eliminate other potential regulators.

\section{MicroRNA target sites obscured by repetitive element insertions}

Our identification of an extensive and uncharacterized 3'UTR for human AID reveals the presence of sequence elements that may confound miR regulatory relationships for other mRNAs. We find that the AID 3'UTR is $\sim 1000 \mathrm{nt}$ longer than previously annotated likely due to mispriming from an internal stretch of 20 adenosines. The full length AID 3'UTR contains a previously unidentified regulatory sequence, an unusually pronounced miR-93 complementarity, (Figure 1) conferring marked repression to targeted transcripts (Figure 2 and Additional File 2, Figure S1). Clearly, this finding agrees with recent suggestions that cryptic miR complementarities may actually be quite common with nearly $50 \%$ of human and mouse 3'UTRs likely extending well beyond their annotated termini $[43,44]$. Since 3'UTRs contain miR binding sites, our analysis of $A I D$ is consistent with the notion that other miR posttranscriptional regulations may have been overlooked because of truncated 3'UTR annotations. This may be particularly pronounced in humans as $5-10 \%$ of all human 3'UTRs contain at least one Alu repeat, a common miR-associated retro-element characterized by a central 10-40 nt adenosine linker $[32,45-51]$.

\section{Conclusions}

In the present study, two human microRNAs, miR-155 and miR-93, were each shown to repress the translation of human AID through interactions with the AID 3'UTR in the MCF-7 breast carcinoma cell line which aberrantly expresses $A I D$. Together, our data suggest that low-level errant $A I D$ expression and subsequent genome damage may be prevented through protective miR-93 and/or miR-155 regulation. In addition, our identification of a miR-93 target site for AID located downstream of an internal adenosine repeat highlights the possibility that other miR regulations may have been overlooked because of truncated 3'UTR annotations.

\section{Additional material}

Additional file 1: Table S1. Oligonucleotide master list. All oligonucleotides were synthesized commercially (Fisher, Operon) at either 25 or 100 nM scale. "pAL" refers to the human miR-517a Alu promoter. "93" and "155" refer to human miRs. "TS" refers to a siRNA or miR target site. ', lowercase indicates imperfect antisense. ${ }^{2}$, AID 3'LR was generated using TS-155F and TS-93R. ${ }^{3}$, designed previously [See reference [32]].

Additional file 2: Figure S1. The AID $3^{\prime} U T R$ is recognized by miRs -93 and $\mathbf{- 1 5 5}$. (A) The AID 3'UTR recognition by miRs -93 and -155 is specific. (A) Diagrams of luciferase reporters. The AID 3'UTR luciferase reporter (TS Intact) and control 3'UTR luciferase reporter (TS Scram) were generated respectively by placing the AID 3'UTR miR -93 and -155 target sites (TS Intact)(with intervening sequences deleted) or an identical sequence in which the target site nucleotides had been scrambled (TS Scram) into a multiple cloning site in the Renilla luciferase $3^{\prime} U T R$ [32]. HSV-TKp, herpes simplex virus thymidine kinase promoter; SV40p, simian virus 40 promoter; FF Luc, firefly luciferase; Ren Luc, Renilla luciferase; pA, poly(A); 155TS, miR-155 target site; 93TS, miR-93 target site; 155SC, miR155 target site scrambled; 93SC, miR-93 target site scrambled. (B) TS Intact is specifically repressed by both miR-93 and miR-155 in 293 transient transfections. Luciferase assays $(n=9)$ of HEK293 lysates after transfection of TS Intact and either pAL-1, pAL-155 or pAL-93.

Transfections were normalized to TS Intact alone. RLU, relative light units. (C) TS Scram is unaffected by co-transfection of pAL-1, pAL-155 or pAL93 in 293 transient transfections. Cotransfections done as in B, except TS Scram replaced TS Intact.

Additional file 3: Figure S2. MicroRNAs miR-93 and miR-155 are widely expressed. RT-PCR of pre-miR template in three different cell lines, HEK293 embryonic kidney cells, MCF-7 breast cancer line, and Ramos Burkitt's lymphoma line. (top) RT-PCR products of the miR-93 locus amplified from indicated cellular RNAs. m, miR-93; $\beta$, Beta actin positive control; - Ctl, no template. (bottom) RT-PCR products of the miR-155 locus amplified from indicated cellular RNAs. Annotations are same as above except $(\mathrm{m})$ denotes pre-miR-155 amplicon. PCR of source RNAs failed, indicating above RT-PCRs templated from the generated cDNA.

Additional file 4: Figure S3. MiR-93 represses endogenous AID in a dose responsive manner. (A) Representative western demonstrating that transient transfection of pAL-93 expression constructs repress endogenous AID protein levels in MCF-7 cells in a dose responsive manner. (B) Relative AID mRNA levels corresponding to replicate transfections performed as in $\mathbf{A}(n=2)$ determined by quantitative PCR.

\section{List of abbreviations}

AID: Activation Induced Deaminase; bp: base pair; ChIP: chromatin immunoprecipitation; EST: expressed sequence tag; HEK: human embryonic kidney; Ig: immunoglobulin; MCF-7: Michigan Cancer Foundation - 7 breast cancer line; miR: microRNA; mRNA: messenger RNA; nt: nucleotide; pAL: Alu promoter; TS: microRNA target site; UTR: mRNA untranslated region; $3^{\prime} \mathrm{LR}$ : 3'UTR luciferase reporter.

\section{Acknowledgements}

The authors would like to recognize support from the School of Biological Sciences and the College of Arts and Sciences at Illinois State University, Phi Sigma Honor Society fellowship to NWH, the National Cancer Institute, National Institutes of Health, 01R15CA137608 to EDL, and the American Cancer Society, Illinois Division, Inc. grant \# 215165 to EDL.

\section{Authors' contributions}

GMB: experimental design, experimentation, and manuscript preparation, $\mathrm{NWH}$ : experimentation, EDL: research design and manuscript preparation. All authors read and approved the final manuscript.

\section{Competing interests}

The authors declare that they have no competing interests.

Received: 6 May 2011 Accepted: 10 August 2011

Published: 10 August 2011

\section{References}

1. de Yebenes VG, Ramiro AR: Activation-induced deaminase: light and dark sides. Trends Mol Med 2006, 12:432-439.

2. Goodman MF, Scharff MD, Romesberg FE: AID-initiated purposeful mutations in immunoglobulin genes. Adv Immunol 2007, 94:127-155.

3. Longerich S, Basu U, Alt F, Storb U: AID in somatic hypermutation and class switch recombination. Curr Opin Immunol 2006, 18:164-174. 
4. Peled JU, Kuang FL, Iglesias-Ussel MD, Roa S, Kalis SL, Goodman MF, Scharff MD: The biochemistry of somatic hypermutation. Annu Rev Immunol 2008, 26:481-511.

5. Vallur AC, Yabuki M, Larson ED, Maizels N: AID in antibody perfection. Cell Mol Life Sci 2007, 64:555-565.

6. Larson ED, Bednarski DW, Maizels N: High-fidelity correction of genomic uracil by human mismatch repair activities. BMC Mol Biol 2008, 9:94

7. Stavnezer J, Guikema JE, Schrader CE: Mechanism and regulation of class switch recombination. Annu Rev Immunol 2008, 26:261-292.

8. Liu M, Schatz DG: Balancing AID and DNA repair during somatic hypermutation. Trends Immunol 2009, 30:173-181.

9. Perez-Duran P, de Yebenes VG, Ramiro AR: Oncogenic events triggered by AID, the adverse effect of antibody diversification. Carcinogenesis 2007, 28:2427-2433.

10. Yamane A, Resch W, Kuo N, Kuchen S, Li Z, Sun HW, Robbiani DF, McBride K, Nussenzweig MC, Casellas R: Deep-sequencing identification of the genomic targets of the cytidine deaminase AID and its cofactor RPA in B lymphocytes. Nat Immunol 2011, 12:62-69.

11. Staszewski O, Baker RE, Ucher AJ, Martier R, Stavnezer J, Guikema JE: Activation-induced cytidine deaminase induces reproducible DNA breaks at many non-lg Loci in activated B cells. Mol Cell 2011, 41:232-242.

12. Liu M, Duke JL, Richter DJ, Vinuesa CG, Goodnow CC, Kleinstein SH, Schatz DG: Two levels of protection for the B cell genome during somatic hypermutation. Nature 2008, 451:841-845

13. Nagaoka H, Tran TH, Kobayashi M, Aida M, Honjo T: Preventing AID, a physiological mutator, from deleterious activation: regulation of the genomic instability that is associated with antibody diversity. Int Immunol 2010, 22:227-235.

14. Okazaki IM, Hiai H, Kakazu N, Yamada S, Muramatsu M, Kinoshita K, Honjo T: Constitutive expression of AID leads to tumorigenesis. J Exp Med 2003, 197:1173-1181.

15. Parsa JY, Basit W, Wang CL, Gommerman JL, Carlyle JR, Martin A: AID mutates a non-immunoglobulin transgene independent of chromosomal position. Mol Immunol 2007, 44:567-575.

16. Zhang W, Bardwell PD, Woo CJ, Poltoratsky V, Scharff MD, Martin A: Clonal instability of $\mathrm{V}$ region hypermutation in the Ramos Burkitt's lymphoma cell line. Int Immunol 2001, 13:1175-1184.

17. Rossi D, Berra E, Cerri M, Deambrogi C, Barbieri C, Franceschetti S, Lunghi M, Conconi A, Paulli M, Matolcsy A, Pasqualucci L, Capello D, Gaidano G: Aberrant somatic hypermutation in transformation of follicular lymphoma and chronic lymphocytic leukemia to diffuse large B-cell lymphoma. Haematologica 2006, 91:1405-1409.

18. Casellas R, Yamane A, Kovalchuk AL, Potter M: Restricting activationinduced cytidine deaminase tumorigenic activity in B lymphocytes. Immunology 2009, 126:316-328.

19. Ramiro AR, Jankovic M, Eisenreich T, Difilippantonio S, Chen-Kiang S, Muramatsu M, Honjo T, Nussenzweig A, Nussenzweig MC: AID is required for c-myc/lgH chromosome translocations in vivo. Cell 2004, 118:431-438.

20. Robbiani DF, Bothmer A, Callen E, Reina-San-Martin B, Dorsett $Y$ Difilippantonio S, Bolland DJ, Chen HT, Corcoran AE, Nussenzweig A, Nussenzweig MC: AID is required for the chromosomal breaks in c-myc that lead to c-myc/lgH translocations. Cell 2008, 135:1028-1038.

21. Endo $Y$, Marusawa H, Chiba T: Involvement of activation-induced cytidine deaminase in the development of colitis-associated colorectal cancers. J Gastroenterol 2011, 46(Suppl 1):6-10.

22. Matsumoto $Y$, Marusawa H, Kinoshita $K$, Niwa $Y$, Sakai $Y$, Chiba T: Upregulation of activation-induced cytidine deaminase causes genetic aberrations at the CDKN2b-CDKN2a in gastric cancer. Gastroenterology 2010, 139:1984-1994.

23. Shinmura K, Igarashi H, Goto M, Tao H, Yamada H, Matsuura S, Tajima M, Matsuda T, Yamane A, Funai K, Tanahashi M, Niwa H, Ogawa H, Sugimura $\mathrm{H}$ : Aberrant Expression and Mutation-Inducing Activity of AID in Human Lung Cancer. Ann Surg Oncol 2011.

24. Babbage G, Ottensmeier CH, Blaydes J, Stevenson FK, Sahota SS: Immunoglobulin heavy chain locus events and expression of activationinduced cytidine deaminase in epithelial breast cancer cell lines. Cancer Res 2006, 66:3996-4000

25. Pauklin S, Sernandez IV, Bachmann G, Ramiro AR, Petersen-Mahrt SK Estrogen directly activates AID transcription and function. J Exp Med 2009, 206:99-111.
26. Lau NC, Lim LP, Weinstein EG, Bartel DP: An abundant class of tiny RNAs with probable regulatory roles in Caenorhabditis elegans. Science 2001, 294:858-862.

27. Lee RC, Ambros V: An extensive class of small RNAs in Caenorhabditis elegans. Science 2001, 294:862-864.

28. Farazi TA, Spitzer Jl, Morozov P, Tuschl T: miRNAs in human cancer. $J$ Pathol 2011, 223:102-115.

29. Dorsett $Y$, McBride KM, Jankovic M, Gazumyan A, Thai TH, Robbiani DF, Di Virgilio M, San-Martin BR, Heidkamp G, Schwickert TA, Eisenreich T, Rajewsky K, Nussenzweig MC: MicroRNA-155 suppresses activationinduced cytidine deaminase-mediated Myc-Igh translocation. Immunity 2008, 28:630-638.

30. Teng G, Hakimpour P, Landgraf P, Rice A, Tuschl T, Casellas R, Papavasiliou FN: MicroRNA-155 is a negative regulator of activationinduced cytidine deaminase. Immunity 2008, 28:621-629.

31. Kluiver J, Haralambieva E, de Jong D, Blokzijl T, Jacobs S, Kroesen BJ, Poppema S, van den Berg A: Lack of BIC and microRNA miR-155 expression in primary cases of Burkitt lymphoma. Genes Chromosomes Cancer 2006, 45:147-153.

32. Borchert GM, Lanier W, Davidson BL: RNA polymerase III transcribes human microRNAs. Nat Struct Mol Biol 2006, 13:1097-1101.

33. Sale JE, Neuberger MS: TdT-accessible breaks are scattered over the immunoglobulin V domain in a constitutively hypermutating B cell line. Immunity 1998, 9:859-869.

34. Landgraf $P$, Rusu $M$, Sheridan $R$, Sewer A, lovino N, Aravin A, Pfeffer S, Rice A, Kamphorst AO, Landthaler $\mathrm{M}$, et al: A mammalian microRNA expression atlas based on small RNA library sequencing. Cell 2007, 129:1401-1414.

35. Ebert MS, Neilson JR, Sharp PA: MicroRNA sponges: competitive inhibitors of small RNAs in mammalian cells. Nat Methods 2007, 4:721-726.

36. Ebert MS, Sharp PA: MicroRNA sponges: progress and possibilities. RNA 2010, 16:2043-2050.

37. Long J, Wang $Y$, Wang W, Chang BH, Danesh FR: Identification of microRNA-93 as a novel regulator of vascular endothelial growth factor in hyperglycemic conditions. J Biol Chem 2010, 285:23457-23465.

38. Petrocca F, Visone R, Onelli MR, Shah MH, Nicoloso MS, de Martino I, \|liopoulos D, Pilozzi E, Liu CG, Negrini M, Cavazzini L, Volinia S, Alder H, Ruco LP, Baldassarre G, Croce CM, Vecchione A: E2F1-regulated microRNAs impair TGFbeta-dependent cell-cycle arrest and apoptosis in gastric cancer. Cancer Cell 2008, 13:272-286.

39. Du L, Schageman JJ, Subauste MC, Saber B, Hammond SM, Prudkin L, Wistuba II, Ji L, Roth JA, Minna JD, Pertsemlidis A: miR-93, miR-98, and miR-197 regulate expression of tumor suppressor gene FUS1. Mol Cancer Res 2009, 7:1234-1243.

40. Fang L, Deng Z, Shatseva T, Yang J, Peng C, Du WW, Yee AJ, Ang LC, He C, Shan SW, Yang BB: MicroRNA miR-93 promotes tumor growth and angiogenesis by targeting integrin-beta8. Oncogene 2011, 30:806-821.

41. Kim YK, Yu J, Han TS, Park SY, Namkoong B, Kim DH, Hur K, Yoo MW, Lee HJ, Yang HK, Kim VN: Functional links between clustered microRNAs: suppression of cell-cycle inhibitors by microRNA clusters in gastric cancer. Nucleic Acids Res 2009, 37:1672-1681.

42. Yeung ML, Yasunaga J, Bennasser Y, Dusetti N, Harris D, Ahmad N, Matsuoka M, Jeang KT: Roles for microRNAs, miR-93 and miR-130b, and tumor protein 53-induced nuclear protein 1 tumor suppressor in cell growth dysregulation by human T-cell lymphotrophic virus 1. Cancer Res 2008, 68:8976-8985.

43. Djebali S, Kapranov P, Foissac S, Lagarde J, Reymond A, Ucla C, Wyss C, Drenkow J, Dumais E, Murray RR, Lin C, Szeto D, Denoeud F, Calvo M, Frankish A, Harrow J, Makrythanasis P, Vidal M, Salehi-Ashtiani K, Antonarakis SE, Gingeras TR, Guigo R: Efficient targeted transcript discovery via array-based normalization of RACE libraries. Nat Methods 2008, 5:629-635.

44. Thorrez L, Tranchevent LC, Chang HJ, Moreau Y, Schuit F: Detection of novel $3^{\prime}$ untranslated region extensions with $3^{\prime}$ expression microarrays. BMC Genomics 2010, 11:205.

45. Borchert GM, Gilmore BL, Spengler RM, Xing Y, Lanier W, Bhattacharya D, Davidson BL: Adenosine deamination in human transcripts generates novel microRNA binding sites. Hum Mol Genet 2009, 18:4801-4807.

46. Hasler J, Samuelsson T, Strub K: Useful 'junk': Alu RNAs in the human transcriptome. Cell Mol Life Sci 2007, 64:1793-1800. 
47. Jurka J, Gentles AJ: Origin and diversification of minisatellites derived from human Alu sequences. Gene 2006, 365:21-26.

48. Lehnert S, Van Loo P, Thilakarathne PJ, Marynen P, Verbeke G, Schuit FC: Evidence for co-evolution between human microRNAs and Alu-repeats. PLoS One 2009, 4:e4456.

49. Mighell AJ, Markham AF, Robinson PA: Alu sequences. FEBS Lett 1997, 417:1-5.

50. Smalheiser NR, Torvik VI: Alu elements within human mRNAs are probable microRNA targets. Trends Genet 2006, 22:532-536.

51. Yulug IG, Yulug A, Fisher EM: The frequency and position of Alu repeats in CDNAs, as determined by database searching. Genomics 1995, 27:544-548.

\section{Pre-publication history}

The pre-publication history for this paper can be accessed here: http://www.biomedcentral.com/1471-2407/11/347/prepub

doi:10.1186/1471-2407-11-347

Cite this article as: Borchert et al:: Repression of human activation induced cytidine deaminase by miR-93 and miR-155. BMC Cancer 2011 $11: 347$

\section{Submit your next manuscript to BioMed Central} and take full advantage of:

- Convenient online submission

- Thorough peer review

- No space constraints or color figure charges

- Immediate publication on acceptance

- Inclusion in PubMed, CAS, Scopus and Google Scholar

- Research which is freely available for redistribution 